\title{
ALGEBRAIC DISTANCE GRAPHS AND RIGIDITY
}

\author{
M. HOMMA AND H. MAEHARA
}

\begin{abstract}
An algebraic distance graph is defined to be a graph with vertices in $E^{n}$ in which two vertices are adjacent if and only if the distance between them is an algebraic number. It is proved that an algebraic distance graph with finite vertex set is complete if and only if the graph is "rigid". Applying this result, we prove that (1) if all the sides of a convex polygon $\Gamma$ which is inscribed in a circle are algebraic numbers, then the circumradius and all diagonals of $\Gamma$ are also algebraic numbers, (2) the chromatic number of the algebraic distance graph on a circle of radius $r$ is $\infty$ or 2 accordingly as $r$ is algebraic or not. We also prove that for any $n>0$, there exists a graph $G$ which cannot be represented as an algebraic distance graph in $E^{n}$.
\end{abstract}

\section{INTRODUCTION}

Let $X$ be a nonempty point set in a Euclidean space, and $D$ a set of real numbers. Let $X(D)$ denote the graph with vertex set $X$ in which two distinct points $x, y$ are adjacent if and only if

$$
|x-y| \in D \text {. }
$$

The graph $X(D)$ is called the $D$-distance graph on $X$. Specifying $D$ in various ways, there arise many interesting graphs. If $D=\{1\}$, we have unit distance graphs, e.g., $[4,9]$. The case $D=[0,1]$ is a generalization of unit interval graphs, e.g. $[7,8,10]$. Letting $D$ be the set of integers, Anning and Erdös [1] proved that if $X(D)$ is a complete graph of infinite order, then all points of $X$ lie on a line. Coloring problems of the real line for various $D$ are discussed in [6].

In this paper we consider the case $D=A$, the set of all algebraic numbers. We call $X(A)$ the algebraic distance graph on $X$. We prove that for a finite set $X$, the algebraic distance graph $X(A)$ is complete if and only if $X(A)$ is a "rigid" graph. Applying this result, we have that if the sides of a convex polygon $\Gamma$, which is inscribed in a circle, are all algebraic numbers, then all diagonals of $\Gamma$ and the radius of the circumcircle of $\Gamma$ are also algebraic numbers.

Concerning the chromatic number of the algebraic distance graph on a circle $C_{r}$ of radius $r$, we have

$$
\chi\left(C_{r}(A)\right)= \begin{cases}\infty & \text { if } r \text { is algebraic }, \\ 2 & \text { otherwise }\end{cases}
$$

Received by the editors August 10, 1988.

1980 Mathematics Subject Classification (1985 Revision). Primary 05C99, 52A37. 
It is not difficult to see that every finite graph $G$ is represented as an algebraic distance graph in Euclidean space. We also prove that for any integer $n>0$, there is a finite graph $G$ which cannot be represented as an algebraic distance graph in Euclidean $n$-space $E^{n}$.

\section{Preliminary Results}

In this section the set $D$ is not specified. Let $\bar{D}=R-D$, the complement of $D$ in the reals. Throughout this paper, $X$ stands for a nonempty point set in Euclidean space. The following lemma is clear.

Lemma 1. A graph $G$ is isomorphic to $X(D)$ if and only if $\bar{G}$ is isomorphic to $X(\bar{D})$, where $\bar{G}$ is the complement of the graph $G$.

Theorem 1. Suppose that both $D$ and $\bar{D}$ contain at least one positive. number. Then every finite simple graph $G$ is isomorphic to $X(D)$ for some $X$.

Proof. Let $v_{1}, \ldots, v_{n}$ be the vertices of $G$, and $A(G)=\left(a_{i j}\right)$ be the adjacency matrix of $G$, i.e., $a_{i j}=1$ or 0 accordingly as $v_{i}, v_{j}$ are adjacent or not. If $t>0$ is a sufficiently large number (which will be specified later), then the matrix $A(G)+t I$ ( $I$ : the identity matrix) is symmetric and positive definite. (Indeed, $t>n$ is sufficient because the minimum eigenvalue of $A(G)$ is greater than $-n$, see, e.g., [14].) Hence it can be represented as the product of an $(n \times n)$-matrix $M$ and its transpose $M^{\prime}$ :

$$
A(G)+t I=M \cdot M^{\prime} .
$$

Let $\bar{v}_{i}$ denote the point in Euclidean $n$-space that corresponds to the $i$ th row of $M$. Then the inner product $\left\langle\bar{v}_{i}, \bar{v}_{j}\right\rangle=t$ if $i=j$, and $=a_{i j}$ if $i \neq j$. Therefore,

$$
\left|\bar{v}_{i}-\bar{v}_{j}\right|^{2}=2 t-2 a_{i j} \text {. }
$$

Now, since both $D$ and $\bar{D}$ contain at least one positive number, there exists a frontier point $c>0$ of $D$. Choose two positive numbers $a \in D, b \in \bar{D}$ sufficiently close to $c$ so that $(1 / a+1 / b)|a-b|<1 / n$. If $a<b$, we put

$$
t=b^{2} /\left(b^{2}-a^{2}\right), \quad \widetilde{v}_{i}=\left(\left(b^{2}-a^{2}\right) / 2\right)^{1 / 2} \bar{v}_{i} .
$$

Then it can easily be verified that $\left|\widetilde{v}_{i}-\widetilde{v}_{j}\right|=a$ if $a_{i j}=1$, and $=b$ if $a_{i j}=0$. Thus the $D$-distance graph on $\left\{\widetilde{v}_{1}, \ldots, \widetilde{v}_{n}\right\}$ is isomorphic to $G$. If $a>b$, then we can prove that there exists a point set $X$ such that $X(\bar{D})$ is isomorphic to $\bar{G}$. Then, applying Lemma 1 , we have the theorem.

Thus every finite graph is represented as $X(A)$ for some $X$. The dimension of the flat spanned by $X$ is denoted by $\operatorname{dim}(X)$. For a graph $G$, let $\operatorname{dim}_{A} G$ denote the minimum value of $\operatorname{dim}(X)$ such that $X(A)$ is isomorphic to $G$. In $\S 6$, we will investigate $\operatorname{dim}_{A} G$.

\section{AlgEBRAIC DISTANCE GRAPHS AND RIGIDITY}

A graph $G$ with vertices in Euclidean space $E^{n}$ is called a graph in $E^{n}$. Thus a graph $G$ in $E^{n}$ is a nonempty subset of $E^{n}$ in which a number of pairs 
are distinguished as the edges. An isotopy of a graph $G$ in $E^{n}$ with vertex set $X$ is a parametrized family of embeddings $f_{t}: X \rightarrow E^{n}, 0 \leq t<\varepsilon$, such that

(1) $f_{0}(x)=x$ for all $x \in X$,

(2) $f_{t}(x)$ is continuous on $t$,

(3) $\left|f_{t}(x)-f_{t}(y)\right|=|x-y|$ for all edges $x y$ to $G$.

A graph $G$ in $E^{n}$ is said to be flexible if there is an isotopy $f_{t}$ of $G$ with

$$
\left|f_{t}(x)-f_{t}(y)\right| \neq|x-y|
$$

for some pair of points $x, y$ of $G$ and some $t$. If $G$ is not flexible, then $G$ is said to be rigid in $E^{n}$. For example, a complete graph in $E^{n}$ is always rigid.

For more information on rigidity and flexibility of graphs, see, e.g., $[2,3,13]$.

The next theorem will relate rigidity to algebraic distance graphs. A system of $(n+1)$ points $p_{i}, i=0, \ldots, n$, is said to be affinely independent if the vectors $\overrightarrow{p_{0} p_{1}}, i=1, \ldots, n$, are linearly independent. Thus $n$ points in $E^{n}$ are affinely independent if and only if they span a hyperplane. The following lemma is clear, and we omit the proof.

Lemma 2. Let $G_{1}, G_{2}$ be two rigid graphs in $E^{n}$ with vertex sets $X_{1}, X_{2}$, respectively. Suppose that $X_{1} \cap X_{2}$ contains a set of $n$ affinely independent points $p_{1}, \ldots, p_{n}$. Then the following holds:

(1) If an isotopy $f_{t}: X_{1} \rightarrow E^{n}$ of $G_{1}$ fixes $p_{i}, i=1, \ldots, n$, then $f_{t}$ fixes all points of $G_{1}$.

(2) If a graph $G$ with vertex set $X_{1} \cup X_{2}$ contains $G_{1}, G_{2}$ as subgraphs, then $G$ is rigid.

Theorem 2. For a finite set $X \subset E^{n}$, the algebraic distance graph $X(A)$ is complete if and only if it is rigid.

To prove this theorem, we need the following proposition concerning algebraic sets, the proof of which will be given in $\S 7$.

Proposition 1. Let $\left\{f_{1}\left(x_{1}, \ldots, x_{N}\right), \ldots, f_{m}\left(x_{1}, \ldots, x_{N}\right)\right\}$ be a collection of polynomials with coefficients in the set $A$ of all real algebraic numbers. Let $V$ denote the real algebraic set defined by $f_{1}=\cdots=f_{m}=0$. Suppose that $p=\left(s_{1}, \ldots, s_{N}\right) \in V$ is an isolated point of $V$. Then $s_{1}, \ldots, s_{N}$ are all algebraic numbers.

Proof of Theorem 2. It is obvious that if $X(A)$ is complete, then it is rigid.

Suppose that $X(A)$ is rigid. Let $k=\operatorname{dim}(X)$. Then without loss of generality, we may assume that $X$ is in Euclidean $k$-space. Let $X=\left\{p_{1}, p_{2}, \ldots, p_{n}\right\}$, and $p_{i}=\left(s_{i, 1}, \ldots, s_{i, k}\right), i=1,2, \ldots, n$. We may further suppose that 
$p_{1}, p_{2}, \ldots, p_{k+1}$ span the $k$-space and

$$
\begin{aligned}
p_{1}= & (0, \ldots, 0), \\
p_{2}= & \left(s_{2,1}, 0, \ldots, 0\right), \\
p_{3}= & \left(s_{3,1}, s_{3,2}, 0, \ldots, 0\right), \\
& \quad \ldots \\
p_{k}= & \left(s_{k, 1}, \ldots, s_{k, k-1}, 0\right) .
\end{aligned}
$$

If $\left|p_{i}-p_{j}\right|$ is an algebraic number, then denote this number by $e_{i j}$, that is, $e_{i j}$ is the "edge length". Now consider the following system of polynomial equations:

$$
\left\{\begin{array}{cc}
\left(x_{i, 1}-x_{j, 1}\right)^{2}+\cdots+\left(x_{i, k}-x_{j, k}\right)^{2}-e_{i j}^{2}=0 & \text { for all "edges" of } X(A), \\
x_{i, i}=\cdots=x_{i, k}=0 \quad & \text { for } i=1, \ldots, k .
\end{array}\right.
$$

These equations define a real algebraic set $V$ in $k n$-space $E^{k n}$, and the point $p=\left(p_{1}, \ldots, p_{n}\right)$ is a point of $V$. For a moment, suppose that $p$ is an isolated point of $V$. Then by Proposition 1, all coordinates of $p$ are algebraic numbers, and hence the distance between any two points of $X$ is an algebraic number; therefore, $X(A)$ is a complete graph.

Thus our remaining task is to show that $p$ is an isolated point of $V$. Suppose, on the contrary, that $V-\{p\}$ contains points arbitrarily close to $p$. Then "the curve selection lemma" (see Milnor [11, p. 25]) asserts that there exists a real analytic curve $x:[0, \varepsilon) \rightarrow V$ with $x(0)=p$ and $x(t) \neq p$ for $t>0$. Denote by $f_{t}\left(p_{i}\right)$ the projection of $x(t) \in E^{k} \times \cdots \times E^{k}=E^{k n}$ onto the $i$ th factor $E^{k}$, that is,

$$
x(t)=\left(f_{t}\left(p_{1}\right), \ldots, f_{t}\left(p_{n}\right)\right) \in E^{k} \times \cdots \times E^{k}=E^{k n} .
$$

Then, since $x(t)(0 \leq t<\varepsilon)$ lies on the algebraic set $V$ defined by $(*)$, $f_{t}: X \rightarrow E^{k}$ is an isotopy of the graph $X(A)$. And since $X(A)$ is rigid, we have

$$
\left|f_{t}\left(p_{i}\right)-f_{t}\left(p_{j}\right)\right|=\left|p_{i}-p_{j}\right| \text { for all } i \neq j .
$$

Furthermore, since the equations $x_{i, i}=\cdots=x_{i, k}=0, i=1, \ldots, k$, hold on the algebraic set $V$, the last $k-i+1$ coordinates of $f_{t}\left(p_{i}\right)$ are zero for $i=1, \ldots, k$, that is,

$$
f_{t}\left(p_{i}\right)=(\underbrace{*, \ldots, *}_{i-1}, \underbrace{0, \ldots, 0}_{k-i+1})
$$

First, we show that $f_{t}$ fixes $p_{i}, i=1, \ldots, k$. This is done by induction on $i$. For $i=1, f_{t}\left(p_{1}\right)=(0, \ldots, 0)$ by $(2)$, whence $f_{t}\left(p_{1}\right)=p_{1}$. Suppose $f_{t}\left(p_{i}\right)=p_{i}$ for $i<j \leq k$. By (2) we may regard $\left\{f_{t}\left(p_{1}\right), \ldots, f_{t}\left(p_{j}\right)\right\}$ as a subset of $(j-1)$-space $E^{j-1}$, and by (1) we may regard $f_{t}$ as an isotopy of the complete graph on $\left\{f_{t}\left(p_{1}\right), \ldots, f_{t}\left(p_{j}\right)\right\}$. Then since $f_{t}$ fixes $p_{1}, \ldots, p_{j-1}$ which are affinely independent, it follows from Lemma 2(1) that $f_{t}\left(p_{j}\right)=p_{j}$. Thus, $f_{t}$ fixes $p_{1}, \ldots, p_{k}$. Now since these $k$ points are affinely independent, 


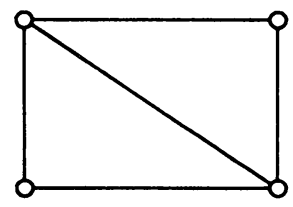

Figure 1

applying Lemma 2(1) again, we have $f_{t}(q)=q$ for all $q$ of $X$. Therefore, $x(t)=p$, a contradiction.

Example 1. Let $G$ be the graph obtained from the complete graph $K_{4}$ by removing one edge (see Figure 1). Since any graph in the plane isomorphic to $G$ is rigid, as is easily seen, $G$ cannot be represented by an algebraic graph in the plane. However, it can be represented in 3-space. (How ?) Hence $\operatorname{dim}_{A} G=3$.

\section{A FEW APplications}

Let $T$ be a triangle with sides $a, b, c$ all algebraic numbers. Then by Heron's formula, the area of $T$ is also algebraic. Let $r$ be the radius of the circumcircle of $T$. Then by the sine law, $c /(\sin \gamma)=2 r$, where $\gamma$ is the angle opposite the side $c$. Hence the area of $T$ is represented as $(1 / 2) a b(\sin \gamma)=$ $a b c /(4 r)$. Therefore the circumradius $r$ of $T$ is also an algebraic number. The next theorem genrealizes this result.

Theorem 3. Let $\Delta$ be an n-dimensional simplex in $E^{n}$ whose sides are all algebraic numbers. Then the radius of the circumsphere of $\Delta$ (i.e., the sphere passing through all vertices of $\Delta$ ) is also an algebraic number.

Proof. Let $r$ be the radius of the circumsphere of $\Delta$. We regard $\Delta$ as a simplex lying on a hyperplane $H$ in $E^{n+1}$. Let $L$ be the line passing through the circumcenter of $\Delta$, and perpendicular to the hyperplane $H$. Let $p, q$ be the two points on $L$, each at distance

$$
\left(m-r^{2}\right)^{1 / 2}
$$

from the circumcenter, where $m$ is a sufficiently large integer. Then the vertices of $\Delta$ and $p, q$, induce together an algebraic distance graph $G$, which is rigid by Lemma $2(2)$. Hence by Theorem $2, G$ is a complete graph, and hence $|p-q|=2\left(m-r^{2}\right)^{1 / 2}$ is algebraic. Therefore, $r$ is algebraic.

By a polygon in the plane, we mean a closed polygonal curve possibly having self-intersections. Let $O$ denote the origin. The area of a polygon $\Gamma=$ $p_{1} p_{2} \cdots p_{n}$ is defined by

$$
\operatorname{area}(\Gamma)=\sum_{i=1}^{n} \varepsilon_{i}\left(\text { area of } \Delta O p_{i} p_{i+1}\right) \quad\left(p_{n+1}:=p_{1}\right),
$$

where $\varepsilon_{i}=+1$ if $O \rightarrow p_{i} \rightarrow p_{i+1} \rightarrow O$ is counter-clockwise, and $\varepsilon_{i}=-1$ otherwise. Note that for a convex polygon, this definition agrees with the usual 
definition of area in absolute value. The winding number wind $(\Gamma)$ of a polygon $\Gamma=p_{1} \cdots p_{n}$ around the origin $O$ is defined by

$$
\operatorname{wind}(\Gamma)=\frac{1}{2 \pi} \sum_{i=1}^{n} \angle p_{i} O p_{i+1},
$$

where $\angle p_{i} O p_{i+1}$ is the signed angle. If $\Gamma$ passes through the origin, then we define $\operatorname{wind}(\Gamma)=0$. For example, a convex polygon $\Gamma$ has winding number \pm 1 if $\Gamma$ contains the origin $O$ inside, and wind $(\Gamma)=0$ otherwise.

For a polygon $\Gamma=p_{1} p_{2} \cdots p_{n}$ in the $x y$-plane in $E^{3}$, the "suspension graph" of $\Gamma$ with poles $q_{1}=(0,0, z), q_{2}=(0,0,-z)$ is the graph with vertices $q_{1}, q_{2}, p_{1}, \ldots, p_{n}$ and edges $p_{i} p_{i+1}, q_{j} p_{i}, i=1, \ldots, n, j=1,2$. The following theorem is a special case of the results of Connelly [5, Theorems 1,2].

Theorem $\mathrm{C}$ (Connelly). Let $\Gamma$ be a polygon in the $x y$-plane in $E^{3}$ and $G$ be the suspension graph of $\Gamma$ with poles $q_{1}=(0,0, z)$ and $q_{2}=(0,0,-z)$. If $G$ is flexible, then

$$
\operatorname{area}(\Gamma)=0 \text { and } \quad \operatorname{wind}(\Gamma)=0 .
$$

If the length of every side of a polygon is an algebraic number, then it will be called an algebraic polygon.

Theorem 4. Let $\Gamma$ be an algebraic polygon inscribed in a circle with center at the origin. If $(\operatorname{area}(\Gamma)$, wind $(\Gamma)) \neq(0,0)$, then the radius $r$ of the circle and the all diagonals of $\Gamma$ are also algebraic numbers.

Proof. Let $p_{1}, \ldots, p_{n}$ be the vertices of the polygon $\Gamma$. We may suppose that the circle lies in the $x y$-plane in $E^{3}$ with center at the origin. Take an integer $m>r$, and put

$$
q_{1}=\left(0,0,\left(m-r^{2}\right)^{1 / 2}\right), \quad q_{2}=\left(0,0,-\left(m-r^{2}\right)^{1 / 2}\right) .
$$

Let $X=\left\{p_{1}, \ldots, p_{n}, q_{1}, q_{2}\right\}$. Then, since $\left|p_{i}-q_{j}\right|=m$, the graph $X(A)$ contains, as a spanning subgraph, the suspension graph of $\Gamma$ with poles $q_{1}, q_{2}$. However, since $(\operatorname{area}(\Gamma), \operatorname{wind}(\Gamma)) \neq(0,0)$, the suspension graph of $\Gamma$ is rigid by Theorem C. Hence $X(A)$ is also rigid, and hence $X(A)$ is complete by Theorem 2 . Therefore, all diagonals of $\Gamma$ and $\left|q_{1}-q_{2}\right|=2\left(m-r^{2}\right)^{1 / 2}$ are algebraic numbers.

We denote by $C_{r}$ the circumference of a circle of radius $r$.

Corollary 1. Let $\Gamma$ be a convex polygon inscribed in a circle $C_{r}$. If the sides of $\Gamma$ are all algebraic numbers, then the radius $r$ and the all diagonals of $\Gamma$ are also algebraic numbers.

Corollary 2. Let $\Gamma$ be an algebraic polygon inscribed in a circle of center $O$ and transcendental radius. Then $\operatorname{area}(\Gamma)=\operatorname{wind}(\Gamma)=0$. 


\section{The Algebraic Distance GRAPH ON A CIRCLE}

In this section we study the (infinite) algebraic distance graph $C_{r}(A)$ on $C_{r}$, the circumference of a circle of radius $r$.

Theorem 5. Suppose $r>0$ is algebraic. Then every connected component of $C_{r}(A)$ is complete.

Proof. Let $p_{0}$ be the center of $C_{r}$. If $p_{1} p_{2} p_{3}$ is a path in the graph $C_{r}(A)$, then $p_{i}, i=0,1,2,3$, induce a rigid algebraic distance graph, whence $\left|p_{1}-p_{3}\right| \in A$, and $p_{1}$ and $p_{3}$ are adjacent in $C_{r}(A)$. From this it follows easily that if two vertices of $C_{r}(A)$ are connected by a path, then the two vertices are adjacent. Therefore, every connected component of $C_{r}(A)$ is complete.

Next, we are going to show that if $r>0$ is transcendental, then $C_{r}(A)$ contains no odd cycle. We begin with a lemma.

Lemma 3. Let $a_{i}, i=1, \ldots, n$, be $n$ distinct algebraic numbers, and let $w$ be a transcendental number such that $w>a_{i}, i=1, \ldots, n$. Then $n$ real numbers $\left(w-a_{i}\right)^{1 / 2}, i=1, \ldots, n$, are linearly independent over the field $A$ of real algebraic numbers.

Proof. Suppose that there is a nontrivial linear combination of the $\left(w-a_{i}\right)^{1 / 2}$ 's such that

$$
b_{1}\left(w-a_{1}\right)^{1 / 2}+\cdots+b_{n}\left(w-a_{n}\right)^{1 / 2}=0, \quad b_{i} \in A
$$

Let $f(x)$ be the function obtained from the left-hand side of the above formula by replacing $w$ by $x$. Then $f(x)$ is a nontrivial algebraic function over $A$ (that is, $f(x)$ is algebraic over the field of rational functions $A(x)$ ). Hence there exists an irreducible polynomial over $A(x)$

$$
F(x, y)=g_{0}(x) y^{k}+g_{1}(x) y^{k-1}+\cdots+g_{k}(x), \quad g_{i}(x) \in A(x),
$$

such that $F(x, f(x))=0$. Note that $g_{k}(x) \neq 0$. Without loss of generality, we may assume that the $g_{i}(x)$ 's are all polynomials over $A$. Now, since $f(w)=0$, we have

$$
0=F(w, f(w))=g_{k}(w),
$$

which is a contradiction since $w$ is transcendental.

Theorem 6. Let $r>0$ be a transcendental number. Then the algebraic distance graph $C_{r}(A)$ contains no odd cycle.

Proof. Without loss of generality, we may assume that the center of the circle $C_{r}$ is at the origin $O$. We show that every cycle of $C_{r}(A)$ is of even order. Consider a cycle of $C_{r}(A)$, and let $\Gamma=p_{1} \cdots p_{n}$ be the corresponding algebraic polygon inscribed in $C_{r}$. We must show that $n$ is even. Let $c_{i}=\left|p_{i}-p_{i+1}\right|$, $i=1, \ldots, n-1, c_{n}=\left|p_{n}-p_{1}\right|$. Then by Corollary 2 , the area of $\Gamma$ is zero, and since the area of the triangle $O p_{i} p_{i+1}$ is $\left(c_{i} / 2\right)\left(r^{2}-\left(c_{i} / 2\right)^{2}\right)^{1 / 2}$, we have

$$
0=\operatorname{area}(\Gamma)=\sum_{i=1}^{i=n} \varepsilon_{i}\left(c_{i} / 2\right)\left(r^{2}-\left(c_{i} / 2\right)^{2}\right)^{1 / 2}
$$


where $\varepsilon=+1$ if the orientation of the triangle $O p_{i} p_{i+1}$ is counter-clockwise, $\varepsilon_{i}=-1$ otherwise. Let $a_{1}, \ldots, a_{m}$ be the distinct numbers in $\left\{c_{1} / 2, \ldots, c_{n} / 2\right\}$ and let $w=r^{2}$. Then, since $\left(w-a_{i}^{2}\right)^{1 / 2}, i=1, \ldots, m$, are linearly independent over $A$ by Lemma 3, it follows from (*) that for each $j(1 \leq j \leq m)$,

$$
0=\sum_{c_{i} / 2=a_{j}} \varepsilon_{i}\left(c_{i} / 2\right)=a_{j} \sum_{c_{i} / 2=a_{j}} \varepsilon_{i} .
$$

Hence the number of subscripts $i$ such that $c_{i} / 2=a_{j}$ is even for every $j$. Therefore $n$ is even.

Corollary 3. If $r>0$ is transcendental, then $C_{r}(A)$ is a bipartite graph.

Corollary 4. The chromatic number of $C_{r}(A)$ is

$$
\chi\left(C_{r}(A)\right)= \begin{cases}\infty & \text { if } r \text { is algebraic }, \\ 2 & \text { otherwise }\end{cases}
$$

The algebraic distance graph on $C_{r}$ with $r$ transcendental has even cycle of arbitrary order $2 n$.

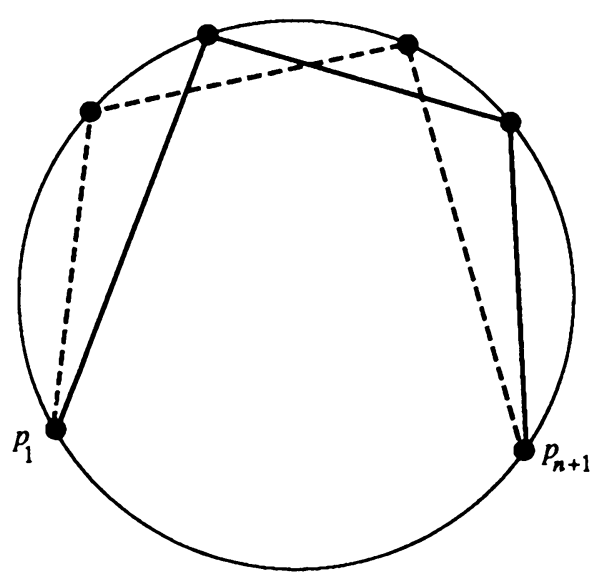

FIGURE 2

Example 2. For any integer $n>1$, the graph $C_{r}(A), r>0$, contains a cycle of order $2 n$. This can be seen as follows: Take a path $P=p_{1} p_{2} \cdots p_{n+1}$ of order $n+1$ in $C_{r}(A)$ (see Figure 2). This is clearly possible. Then reflect this path with respect to the perpendicular bisector of the line segment $p_{1} p_{n+1}$ (in Figure 2 , the reflected path is indicated by dotted line). These two paths together make a cycle of order $2 n$. Of course we must choose a path $P$ so that $P$ and its reflection share only two vertices in common.

Theorem 7. For any $r>0$, the graph $C_{r}(A)$ is disconnected.

Proof. First suppose that $r$ is an algebraic number. Let $p, q \in C_{r}$ be two points such that $|p-q|$ is a transcendental number. Then by Theorem 5 , 
$p$ and $q$ cannot belong to the same component of $C_{r}$. Hence $C_{r}(A)$ is disconnected. Now suppose that $r$ is transcendental and $C_{r}(A)$ is connected. Let $P=p_{1} p_{2} \cdots p_{n}$ be a path of minimum order that connects a pair of diametrically opposite points. Since the diameter $2 r$ is transcendental, $n$ is greater than 2. Let $Q=q_{1} q_{2} \cdots q_{n}$ be the path obtained by rotating $P$ around the center $O$ of $C_{r}$ through the angle $\pi$. Then $q_{1}=p_{n}$ and $q_{n}=p_{1}$. Since the path $P$ is a path of minimum order that connects $p_{1}, p_{n}$, the paths $P, Q$ share only two points in common. Note that

$$
\angle p_{1} O p_{2}+\cdots+\angle p_{n-1} O p_{n}=\angle q_{1} O q_{2}+\cdots+\angle q_{n-1} O q_{n}=(2 m+1) \pi
$$

for some integer $m$. Let $\Gamma$ be the algebraic polygon

$$
p_{1} p_{2} \cdots p_{n-1} q_{1} q_{2} \cdots q_{n-1} \text {. }
$$

Then, $\operatorname{wind}(\Gamma)=(2 m+1) \neq 0$, which contradicts Corollary 2 .

\section{THE MINIMUM DIMENSIONAL REPRESENTATIONS}

First note that if $H$ is an "induced" subgraph of $G$, then $\operatorname{dim}_{A} H \leq \operatorname{dim}_{A} G$. This is not the case for a subgraph. For example, $\operatorname{dim}_{A} K_{4}=1$, but $\operatorname{dim}_{A}\left(K_{4}\right.$ - one edge $)=3$ as already seen.

Theorem 8. For a complete bipartite graph $K(m, n)$ with order $m+n \geq 3$, we have $\operatorname{dim}_{A} K(m, n)=2$.

Thus, for any $m, n>0$, there exists a flexible graph in the plane which is isomorphic to $K(m, n)$.

Proof. It is clear that $\operatorname{dim}_{A} K(m, n)>1$ for $m+n \geq 3$. We represent $K(m, n)$ as an algebraic distance graph in the plane $E^{2}$. Let $p_{i}=\left((i+\pi)^{1 / 2}, 0\right), i=$ $1, \ldots, m ; q_{j}=\left(0,(j+3-\pi)^{1 / 2}\right), j=1, \ldots, n$. Then $\left|p_{i}-q_{j}\right|$ is algebraic, but $\left|p_{i}-p_{j}\right|,\left|q_{i}-q_{j}\right|$ are not algebraic unless $i=j$. Hence the algebraic distance graph on $\left\{p_{1}, \ldots, p_{m}, q_{1}, \ldots, q_{n}\right\}$ is isomorphic to $K(m, n)$.

Now there arises a question: as a function on finite graphs $G$, is $\operatorname{dim}_{A} G$ unbounded?

Lemma 4. Let $W, X, Y, Z$ be four sets in Euclidean space such that

(1) $Z=X \cup Y, W=X \cap Y$,

(2) $W(A), X(A), Y(A)$ are complete, but $Z(A)$ is not complete, and

(3) $\operatorname{dim}(W) \geq i-2$.

Then $\operatorname{dim}(Z) \geq i$, and $\max \{\operatorname{dim}(X), \operatorname{dim}(Y)\} \geq i-1$.

Proof. By (3), there exist $i-1$ affinely independent points in $W$. If $Z$ is contained in $(i-1)$-space $E^{i-1}$, then by Lemma 2(2), $Z(A)$ must be rigid and hence complete, a contradiction. Therefore, $\operatorname{dim}(Z) \geq i$. Since $\operatorname{dim}(W) \geq$ $i-2$, there must be a point $z$ in $Z$ such that $\operatorname{dim}(W \cup\{z\}) \geq i-1$. Hence $\max \{\operatorname{dim}(X), \operatorname{dim}(Y)\} \geq i-1$. 
Theorem 9. Denote by $G_{n}$ the complete n-partite graph $K(2, \ldots, 2)$. Then $\operatorname{dim}_{A} G_{n} \geq n$.

Thus, $\operatorname{dim}_{A} G$ is unbounded as a function on finite graphs $G$.

Proof. Let $U_{n}$ be a point set such that $U_{n}(A)$ is isomorphic to $G_{n}$. Then, corresponding to the nested sequence of induced subgraphs

$$
G_{2} \subset G_{3} \subset \cdots \subset G_{n-1} \subset G_{n}
$$

there is a nested sequence of subsets

$$
U_{2} \subset U_{3} \subset \cdots \subset U_{n-1} \subset U_{n}
$$

such that $U_{i}(A)$ is isomorphic to $G_{i}$. Then $\left|U_{i+1}-U_{i}\right|=2$. We claim that for each $i=2, \ldots, n, U_{i}$ contains subsets $W_{i}, X_{i}, Y_{i}, Z_{i}$ satisfying the conditions of Lemma 4. This is proved by induction on $i$. For $i=2$, take a path $p_{1} p_{2} p_{3}$ in $U_{2}(A)$, and let

$$
W_{2}=\left\{p_{2}\right\}, \quad X_{2}=\left\{p_{1}, p_{2}\right\}, \quad Y_{2}=\left\{p_{2}, p_{3}\right\}, \quad Z_{2}=\left\{p_{1}, p_{2}, p_{3}\right\} .
$$

Then these four sets satisfy the conditions. Suppose that $U_{i}$ contains subsets $W_{i}, X_{i}, Y_{i}, Z_{i}$ satisfying (1)-(3). We may suppose $\operatorname{dim}\left(X_{i}\right) \geq i-1$. Let $\{x, y\}=U_{i+1}-U_{i}$. Then the algebraic distance graphs induced by $X_{i} \cup\{x\}$ and by $X_{i} \cup\{y\}$ are both complete. Hence letting

$$
W_{i+1}=X_{i}, \quad X_{i+1}=X_{i} \cup\{x\}, \quad Y_{i+1}=X_{i} \cup\{y\}, \quad Z_{i+1}=X_{i} \cup\{x, y\},
$$

we have the $(i+1)$-case.

Therefore $\operatorname{dim}\left(U_{n}\right) \geq \operatorname{dim}\left(Z_{n}\right) \geq n$.

Example 3.

$$
\operatorname{dim}_{A} G_{n}=\operatorname{dim}_{A} K \underbrace{(2, \ldots, 2)}_{n}=n \text { for } n \leq 4
$$

Proof. We show only the case $n=4$. In the plane $E^{2}$, take four points $p_{i}, i=1,2,3,4$, on the circle of radius $\pi$ centered at the origin such that $\left|p_{1}-p_{2}\right|,\left|p_{2}-p_{3}\right|,\left|p_{3}-p_{4}\right|,\left|p_{4}-p_{1}\right|$ are all algebraic, see Example 2. Then by Theorem $3,\left|p_{2}-p_{4}\right|$ and $\left|p_{1}-p_{3}\right|$ are transcendental numbers. Take four points $q_{i}, i=1,2,3,4$, with similar property on the circle of, this time, radius $\left(m-\pi^{2}\right)^{1 / 2}$, centered at the origin, where $m$ is a sufficiently large integer. Since the radius $\left(m-\pi^{2}\right)^{1 / 2}$ is transcendental, it also follows from Theorem 3 that $\left|q_{2}-q_{4}\right|$ and $\left|q_{1}-q_{3}\right|$ are transcendental. Now, in $E^{4}=E^{2} \times E^{2}$, let $X$ be the set of eight points

$$
\begin{array}{llll}
\left(p_{1}, 0\right), & \left(p_{2}, 0\right), & \left(p_{3}, 0\right), & \left(p_{4}, 0\right), \\
\left(0, q_{1}\right), & \left(0, q_{2}\right), & \left(0, q_{3}\right), & \left(0, q_{4}\right) .
\end{array}
$$

Then it can be easily verified that $X(A)$ is isomorphic to $G_{4}$. 


\section{Proof of Proposition 1}

As usual, the symbols $Q, R, C$ stand for the set of rational numbers, the set of real numbers, the set of complex numbers, respectively. The algebraic closure of the field $Q$ is denoted by $\bar{Q}$. Thus, $A=\bar{Q} \cap R$.

Let $\mathfrak{P}$ be the prime ideal of $A\left[x_{1}, \ldots, x_{N}\right]$ defined as the kernel of the $A$-algebra homomorphism

$$
\begin{gathered}
A\left[x_{1}, \ldots, x_{N}\right] \rightarrow R, \\
x_{i} \rightarrow s_{i}
\end{gathered}
$$

where $s_{i}$ is the $i$ th coordinate of the isolated point $p=\left(s_{1}, \ldots, s_{N}\right)$ of the algebraic set $V$. Let us denote by $F(\mathfrak{P})$ the quotient field $A\left[x_{1}, \ldots, x_{N}\right] / \mathfrak{P}$. Since $A \subset F(\mathfrak{P}) \subset R$, the field $A$ is algebraically closed in $F(\mathfrak{P})$, that is, $F(\mathfrak{P})$ is a regular extension of $A$. Hence the ideal $\mathfrak{P}$ is absolutely prime; that is, for every extension $K$ of $A$, the ideal $\mathfrak{P} \cdot K\left[x_{1}, \ldots, x_{N}\right]$ is prime (see [16, Chapter VII, Theorem 39]).

For each field $K=\bar{Q}, R, C$, let $W_{K}$ denote the algebraic set in $K^{N}$ defined by the ideal $\mathfrak{P} \cdot K\left[x_{1}, \ldots, x_{N}\right]$. Then $p \in W_{R} \subset W_{C}$, and since $W_{C}$ is defined over $A$ and $\bar{Q}$ is the algebraic closure of $A$, we have

$$
\varnothing \neq W_{\bar{Q}} \subset W_{C}
$$

Since $\mathfrak{P}$ is absolutely prime,

$$
W_{C} \text { is an irreducible algebraic set in } C^{N} \text {. }
$$

Suppose, for a moment, that the algebraic dimension, $\operatorname{dim} W_{C}$, of $W_{C}$ is equal to zero. Then, since $W_{C}$ is irreducible, we have $W_{C}=\{p\}$, and hence by (1), $W_{\bar{Q}}=\{p\}$, i.e., $p=\left(s_{1}, \ldots, s_{N}\right) \in \bar{Q}^{N}$.

Now we show that $\operatorname{dim} W_{C}=0$. Suppose, on the contrary, $\operatorname{dim} W_{C}>0$. In this case, any isolated point of $W_{R}$ is a singular point of $W_{C}$. This fact can be proved by using the implicit function theorem; see [15, Chapter II, 2.3] for details. Then, since $p \in W_{R} \subset V, p$ is an isolated point of $W_{R}$, and hence $p$ is a singular point of $W_{C}$. Let

$$
g_{1}, \ldots, g_{M} \in A\left[x_{1}, \ldots, x_{N}\right]
$$

be a system of generators of $\mathfrak{P}$ and let $r$ be the rank of the $M \times A$ yiatrix $\left(\partial g_{i} / \partial x_{j}\right)$ evaluated on $W_{C}$. Then, since $p$ is a singular point of $W_{C}$, the values of all $r \times r$ minors of the matrix $\left(\partial g_{i} / \partial x_{j}\right)$ at $p$ are zero. Now, by the definitions of $\mathfrak{P}$ and $W_{C}$, we have that

$$
g \in A\left[x_{1}, \ldots, x_{N}\right], g(p)=0 \text { implies that } g=0 \text { on } W_{C}
$$

(i.e., $p$ is an " $A$-generic point" in the sense of Mumford [12]). Therefore, all $r \times r$ minors vanish on $W_{C}$, a contradiction. 


\section{REFERENCES}

1. N. H. Anning and P. Erdös, Integral distances, Bull. Amer. Math. Soc. 51 (1945), 598-600.

2. L. Asimov and B. Roth, The rigidity of graphs, Trans. Amer. Math. Soc. 245 (1978), 279289.

3.

4. K. B. Chilakamarri, Unit-distance graphs in rational $n$-space, Discrete Math. 69 (1988), 213-218.

5. R. Connelly, The rigidity of suspensions, J. Differential Geom. 13 (1978), 399-408.

6. K. B. Eggleion, P. Erdös and D. K. Skilton, Coloring the real line, J. Combin. Theory Ser. B 39 (1985), 86-100.

7. P. C. Fishburn, On the sphericity and cubicity of graphs, J. Combin. Theory Ser. B 35 (1983), 309-318.

8. $\because$ Frankl and $\mathrm{H}$. Maehara, The Johnson-Lindenstrauss lemma and the sphericity of some graphs, J. Combin. Theory Ser. B 44 (1988), 355-362.

9. H. Maehara, Note on induced subgraphs of the unit distance graph $E^{n}$, Discrete Comput. Geom. 4 (1989), 15-18.

10. __ Space graphs and sphericity, Discrete Appl. Math. 7 (1984), 55-64.

11. J. Milnor, Singular points of complex hypersurfaces, Ann. of Math. Stud., no. 61, Princeton Univ. Press, Princeton, N. J., 1968.

12. D. Mumford, Algebraic geometry. I.-Complex projective geometry, Springer-Verlag, New York, 1976.

13. B. Roth, Rigid and flexible frameworks, Amer. Math. Monthly 88 (1981), 6-21.

14. A. J. Schwenk and R. J. Wilson, On the eigenvalue of a graph, Selected Topics in Graph Theory (edited by L. W. Beineke and R. J. Wilson), Academic Press, New York, 1978.

15. I. R. Shafarevich, Basic algebraic geometry, Springer-Verlag, New York, 1974.

16. O. Zariski and P. Samuel, Commutative algebra, Van Nostrand, Princeton, N. J., 1959.

Department of Mathematics, Ryukyu University, Nishihara, Okinawa, Japan 